\title{
News and Reports
}

News items on synchrotron radiation science are published by the Journal of Synchrotron Radiation. Full or partial inclusion is subject to the approval of the Main Editors, to whom all correspondence should be sent.

\section{J. Synchrotron Rad. (1999). 6, 943-944}

\section{The Canadian Light Source}

On 31 March 1999 the Canada Foundation for Innovation (CFI) approved a \$56.4 million contribution to build the Canadian Light Source (CLS) synchrotron project at the University of Saskatchewan, Canada. This decision gives the go-ahead to the $\$ 173.5$ million national facility, the largest scientific project in a generation to be built in Canada.

The CLS represents an unprecedented level of collaboration in Canada among different levels of governments, univer- sities and industry. The project marks the first ever federal-provincial-civic partnership with a university to build a major research project in Canada. In addition to the University of Saskatchewan, 18 other universities have endorsed the project.

The CLS will be built around the existing Saskatchewan Accelerator Laboratory (SAL) on the University of Saskatchewan campus. The state-of-theart facility is expected to begin operation in 2003, and will be owned and operated by the University of Saskatchewan for the various stakeholders. With a Board of Directors having representation from various funding partners, the management structure will emphasize the facility's unique national character and its focus on serving users. The National Research Council (NRC) will work with the University of Saskatchewan to assure that the CLS is managed as a national facility.

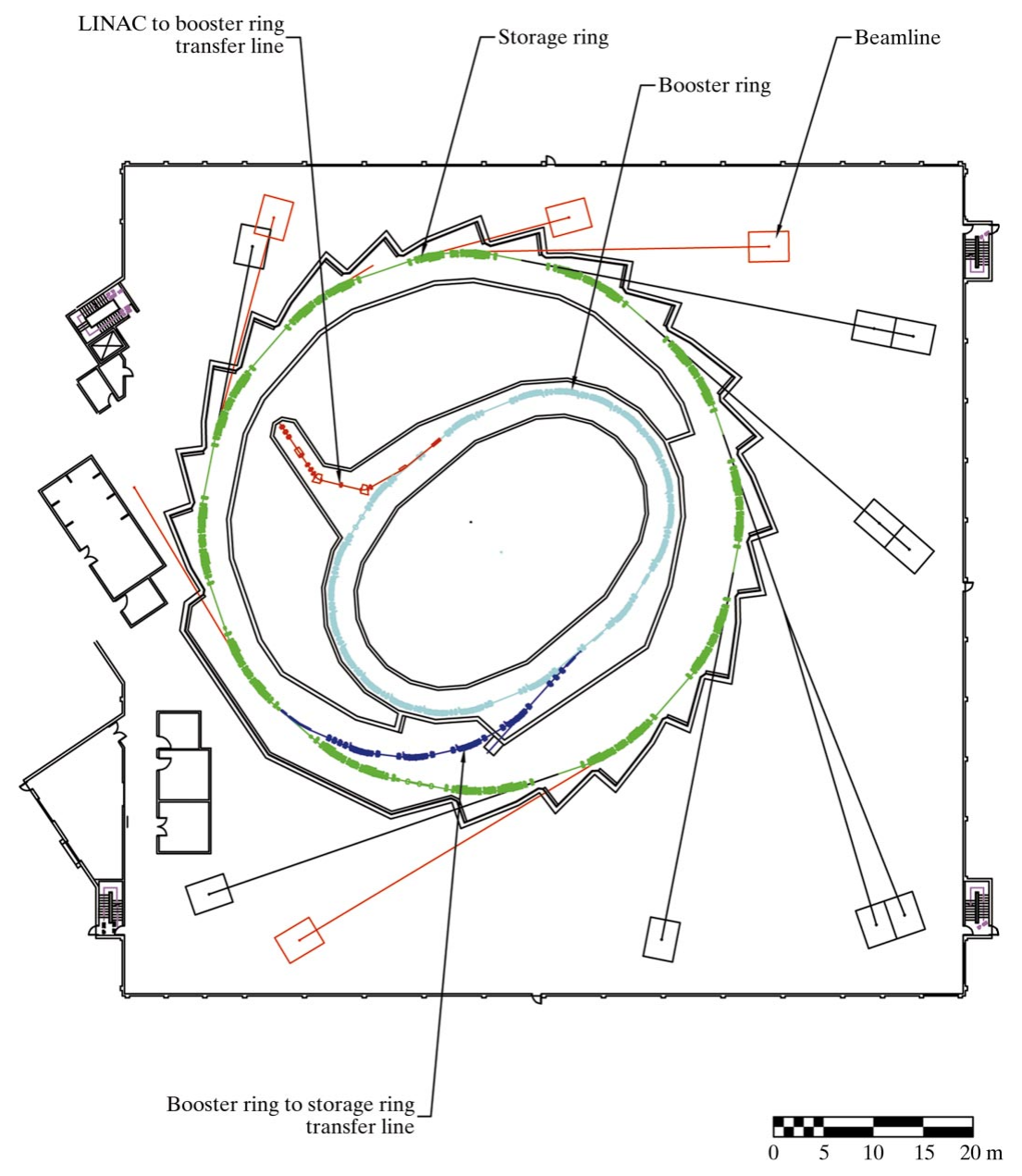

Figure 1

CLS floor plan. The attachment to the existing building is not shown.
The CLS will be of major use for materials research by scientists from all parts of Canada, including physicists, chemists, biologists and those from many other fields. It will also be an important training ground for students.

The total value of the CLS project is $\$ 173.5$ million - a $\$ 140.9$ million cash portion and \$32.6 million in kind contributions, which includes the existing accelerator laboratory and three University of Western Ontario beamlines (scientific workstations) which are to be moved from a facility in Madison, Wisconsin.

The CFI, funded by the Government of Canada, is providing $40 \%$ of the $\$ 140.9$ million in capital costs; other federal departments are contributing another $20 \%$ ( $\$ 28.3$ million). The Government of Saskatchewan will contribute $\$ 25$ million, the University of Saskatchewan $\$ 7.3$ million, the City of Saskatoon \$2.4 million, SaskPower Corporation $\$ 2$ million and the Universities of Alberta and Western Ontario $\$ 300000$ each. A further $\$ 19$ million will flow from other provinces, universities and industry to build beamlines.

The Government of Canada has committed itself to providing a significant portion of the $\$ 13.9$ million $(\sim 55 \%)$ in annual operating costs through agencies such as the Natural Sciences and Engineering Research Council (NSERC), the NRC and the Medical Research Council. The remaining operating costs will be covered by user fees, the University of Saskatchewan and other sources as required.

Preparatory design work by the SAL staff is almost complete. Tenders will be sent out for construction work in the coming months.

CFI President David Strangway said, "The Canada Foundation for Innovation is pleased to be a major funding partner in this project. By announcing our contribution of $\$ 56.4$ million, we are marking a new era for scientific research in Canada. The success of this project can only be made possible thanks to an extraordinary level of commitment and cooperation between the University of Saskatchewan and its partners from the public and private sectors. As a result, researchers will be able to undertake projects that will have a profound impact on many aspects in the lives of Canadians." 


\section{The UK's new synchrotron facility}

The Wellcome Trust has committed $£ 110$ million to the project of building a new synchrotron for the UK research community. Synchrotrons are widely used in science; in biomedical research they are primarily used to determine the structure of large complex molecules such as proteins.

The project will be funded by the Wellcome Trust and also the Office of Science and Technology (OST), which has committed $£ 35$ million to the project over the next three years. The Trust and the OST are currently discussing various options for the specification of the new facility, i.e. how it will be run and where it will be located. Information from existing modern synchrotron facilities around the world is being gathered, and consultations within the UK research community are also being conducted.

The Wellcome Trust sees this project as an exciting opportunity to provide the UK scientific community with a major new state-of-the-art facility. The Trust is keen to work closely with synchrotron users and the Research Councils to develop the project, as these are the main stakeholders in the project.

\section{Coherence workshop report}

Coherence of electromagnetic radiation at optical wavelengths has been known about since the last century, but it is only recently that many experiments have been performed exploiting the coherence of $\mathrm{X}$ ray synchrotron radiation (Hu et al., 1998; Lang et al., 1999). The production and preservation of coherence in beamline optics has not been a major consideration in the design of third-generation synchrotron sources because the possibi- lities of using the coherence have not been appreciated until recently. A one-day meeting chaired by Colin Norris was held at Daresbury Laboratory on 18 May 1999 to discuss the potential applications of coherence and how the proposed UK third-generation storage ring, DIAMOND, could be designed with experiments that exploit coherent radiation in mind.

The meeting included two speakers working at third-generation synchrotron sources, Jose Baruchel from the ESRF and Malcolm Howells from the ALS, and also Andrew Lang from Bristol University, who has performed coherence experiments at the SRS.

David Laundy provided an introduction to coherence and showed that high coherence length is possible with a lowemittance synchrotron source. Jose Baruchel then described work being carried out at the ESRF, emphasizing the connection between coherent flux and the brilliance of radiation and that increase in coherent length can only be achieved at the expense of photon flux. Coherent scattering of radiation depends on the real correction to the refractive index, which for X-rays is a factor 10-1000 times larger than the imaginary correction, and therefore coherent radiation can give much higher contrast than absorption imaging. Speckle interferometry experiments have been carried out at the ESRF, and the speaker talked about the exciting prospect of performing X-ray photon spectroscopy in which the time correlations of speckles are used to study dynamics in new time and length regimes. Imaging experiments are now progressing towards phase reconstruction where images taken at different distances are used to reconstruct a phase image of the object. This could be done in conjunction with tomographic reconstruction and then it would be possible to make three-dimensional images of objects which show only poor absorption contrast.

The meeting then heard from Andrew Lang on work that he had carried out on station 7.6 at Daresbury on Fresnel biprism fringes. He showed how such images could be used to measure the coherent properties of the beam and to quantify the degradation of coherence due to beam instability.

The final talk was given by Malcolm Howells, who outlined the description of coherence using radiation modes. $\mathrm{He}$ emphasized the importance of stability of the experiment to take advantage of the coherence of the beam, and then described work being carried out at the ALS on Fourier transform spectroscopy and holography in the soft X-ray regime and other imaging techniques.

In the discussion that followed, many diverse applications of coherence were discussed. There was agreement on the importance of designing X-ray beamlines in order to deliver a given beam coherence and on the need for development of detectors with better efficiency, spatial resolution and readout speed. While with low-emittance synchrotrons coherence is unavoidable, it can be exploited to open up many new avenues of science.

\section{References}

Hu, Z. H., Thomas, P. A., Snigirev, A., Snigireva, I., Souvorov, A., Smith, P. G. R. Ross, G. W. \& Teat, S. (1998). Nature (London), 392, 690-693.

Lang, A. R. \& Makepeace, A. P. W. (1999). J. Synchrotron Rad. 6, 59-61.

David Laundy Daresbury Laboratory 